\title{
Prediction-based trajectory tracking of External Gas Recirculation for turbocharged SI Engines
}

\author{
Delphine Bresch-Pietri, Thomas Leroy, Jonathan Chauvin, Nicolas Petit
}

\begin{abstract}
This paper addresses the trajectory tracking problem of intake burned gas rate for Spark Ignited engines. We propose a simple linear time-varying input delay model of this dynamics, where the delay is represented by an implicit integral equation involving the past values of the input. We extend some recent results from the literature to design a novel predictor-based controller and compare the merits of the proposed technique with the ones of previous works from the literature. Simulation results stress the relevance of this preliminary work and directions of future work are provided.
\end{abstract}

\section{INTRODUCTION}

Lately, downsizing (reduction of the engine size) has appeared as a major way for SI engines to achieve the still more stringent environmental requirements. Such downsized engine can reach high levels of performance and driveability, provided they are equipped with direct injection, turbocharger and Variable Valve Timing (VVT) actuators [21]. Such a setup is represented in Fig. 1.

On such engines, one major control issue is the prevention of the knock phenomenon, an unwanted self-ignition of the mixture which could appear at hight load (see [7]). One of the considered strategies to use exhaust gas recirculation (EGR) through a low-pressure circuit, represented in Fig. 1 : burned gases are picked up downstream of the catalyst and mixed with fresh air upstream of the compressor. The amount of reintroduced burned gases is controlled by the EGR Valve.

The addition of exhaust gas into the mixture leads to an increase of the auto-ignition delay : intermixing the incoming air with recirculated exhaust gas dilutes the mixture with inert gas, increases its specific heat capacity and consequently lowers the peak combustion temperature. Then, the effect of EGR is a prevention of knock effect which leads to potential substantial improvements of combustion efficiency [?].

In the same time, during tip-outs, the presence of burned gases in the intake manifold seriously impacts the combustion process and the engine may stall. For this reason, an accurate advanced combustion control, based on the estimation and the control of the intake burned gas rate, is unquestionably necessary.

Nevertheless, the relative long distance between the compressor and the inlet manifold leads to a significant transport

D. Bresch-Pietri (corresponding author) is a $\mathrm{PhD}$ Candidate in Mathematics and Control at MINES ParisTech, 60, Bd St-Michel, 75272 Paris, Cedex 06, France. Email : delphine.bresch-pietri@mines-paristech.fr

T. Leroy and J. Chauvin are with the Département Contrôle, Signal et Système in IFP Energies Nouvelles, 1 et 4 Avenue du Bois Préau, 92852 Rueil Malmaison, France

N. Petit is with the Centre Automatique et Systèmes, Unité Mathématiques et Systèmes at MINES ParisTech, 60 Bd St Michel, 75272 Paris, France delay, up to several seconds. Further, this delay depends on the gas flow rate and then on the manipulated actuator, which considerably increases the complexity of the control task. This may explain why this SI engine control problem has surprisingly barely been studied in the literature ${ }^{1}$.

In this paper, we propose to design a prediction-based control law, accounting for this time-varying delay and its dependency on the history of the input.

The robust stability of systems with time-varying delay in the input have been widely studied lately : using either a Lyapunov-Razumikhin function or a Lyapunov-Krasovskii functional, delay-dependent stability criteria are obtained under the form of Linear Matrix Inequalities (LMIs) [9], [10], [14], [22]. The "memoryless" controllers employed in such approaches are relatively easy to implement. Yet, to improve closed-loop dynamic performance, one could prefer to use a predictor-based control law [2], [23], [28] aiming at compensating the delay via a distributed delay of infinite dimension. Such techniques, which are bitterly used for a constant input time-delay (see for instance [13], [16], [24], [25] or [8], [27] and the reference therein) are less popular for time-varying ones. Recently, some were developed in such a framework in [30], where substantial LMIs have to be checked, and in [17], where the invertibility of a certain delay-operator is assumed.

Here, we follow the overture proposed in [15] and [17] to analyze the stability of linear input time-delay systems. The new techniques proposed there have been developed in [3], [4], [5], [18] to address uncertainties toward constant input time-delays in various automotive contexts. Here, we extend these tools to linear systems with time-varying delay in the input, similarly to what was done in [17], and explicitly relate the obtained result with the regulation of the intake burned gas rate for a turbocharged SI engine. These are the main contributions of the paper.

The paper is organized as follows. In Section II, we present a model of the intake burned gas rate dynamics, expressing the considered system as a linear input-delay systems with an input-dependent delay. Then, in Section III, we design a prediction-based control law for such systems and develop the corresponding convergence proof. Finally, numerical results and directions of future work are discussed in Section IV.

\footnotetext{
${ }^{1}$ On the contrary, numerous works focus on this problem for Diesel engine. However, as context and challenges are substantially different, it results into a different control framework (see [1], [29], [31] and the reference therein for more details).
} 


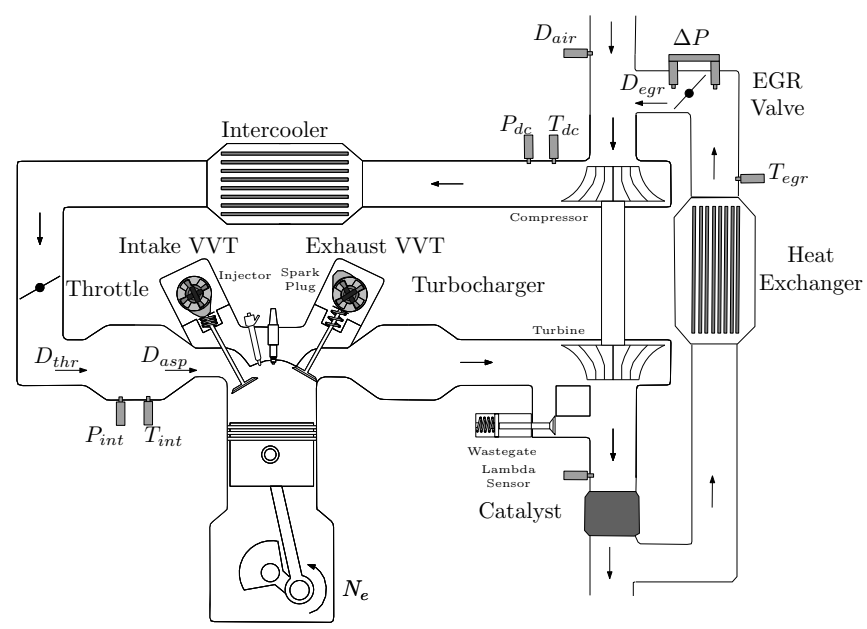

Fig. 1. Scheme of a turbocharged SI engine equipped with direct injection, VVA and a Low-Pressure EGR loop. The $\Delta P$ sensor located at the EGR valve gives an indirect measurement of the EGR mass flow rate $D_{\text {egr }}$ (see Appendix A).

\section{Model Definition}

Consider the airpath of a turbocharged SI engine equipped with intake throttle, waste gate, dual independent VVT actuators and a low-pressure external gas recirculation (EGR) loop as depicted in Fig. 1. Notations presented in Fig. 1 and used below are summarized in Table II.

We focus on the control of the amount of externally recirculated burned gas into the intake manifold. Such a control is realized thanks to the EGR flow rate through the EGR valve, $D_{e g r}$. As this actuator is spatially distant from the intake manifold, it introduces a time-varying transport delay. Assuming that the mixing into the volume downstream the EGR valve is instantaneous (i.e. neglecting the dilution dynamics in this volume), the burned gas rate at this point can be expressed as

$$
u(t)=\frac{D_{e g r}(t)}{D_{\text {air }}(t)+D_{\text {egr }}(t)}
$$

where $D_{a i r}$ is measured and $D_{e g r}$ is directly related to the measured differential of pressure $\Delta P$ (this relation is described in Appendix). Further, we neglect the mixing during the flow transport, namely, following the plug-flow assumption,

$$
L_{P}=\int_{t-\tau(t)}^{t} v_{g a s}(s) d s
$$

where $L_{P}$ represents the pipe length from the EGR-valve down to the intake manifold and $v_{\text {gas }}$ account for the gas speeds. Finally, the mixing dynamics into the intake manifold can be expressed with the composition balance equation ${ }^{2}$

$$
\dot{x}(t)=\alpha\left[-D_{a s p} x(t)+D_{t h r} u(t-\tau(t))\right]
$$

where $x$ represents the externally recirculated burned gas rate into the intake manifold and $\alpha=\frac{r T_{\text {int }}}{P_{\text {int }} V_{\text {int }}}$ is known, as both

\footnotetext{
${ }^{2}$ This balance equation assumes that the EGR circuit is filled only with

\begin{tabular}{|c|c|c|}
\hline Symbol & Description & Unit \\
\hline$D_{\text {air }}$ & Air mass flow rate upstream the compressor & $\mathrm{kg} / \mathrm{s}$ \\
\hline$D_{e g r}$ & EGR mass flow rate through the EGR valve & $\mathrm{kg} / \mathrm{s}$ \\
\hline$D_{t h r}$ & Mass flow rate through the throttle & $\mathrm{kg} / \mathrm{s}$ \\
\hline$D_{a s p}$ & Aspirated mass flow rate & $\mathrm{kg} / \mathrm{s}$ \\
\hline$T_{\text {int }}$ & Intake manifold temperature & $\mathrm{K}$ \\
\hline$P_{\text {int }}$ & Intake manifold pressure & $\mathrm{Pa}$ \\
\hline$T_{d c}$ & Temperature downstream the compressor & $\mathrm{K}$ \\
\hline$P_{d c}$ & Pressure downstream the compressor & $\mathrm{Pa}$ \\
\hline$T_{\text {atm }}$ & Atmospheric temperature & $\mathrm{K}$ \\
\hline$P_{\text {atm }}$ & Atmospheric pressure & $\mathrm{Pa}$ \\
\hline$r$ & Specific ideal gas constant & $\mathrm{J} / \mathrm{kg} / \mathrm{K}$ \\
\hline$N_{e}$ & Engine speed & $\mathrm{rpm}$ \\
\hline$L_{P}$ & $\begin{array}{l}\text { Pipe length from the EGR valve down } \\
\text { to the intake manifold }\end{array}$ & $\mathrm{m}$ \\
\hline$\theta_{e g r}$ & EGR Valve Position & $\%$ \\
\hline$m_{\text {air }}$ & Aspirated air mass & $\mathrm{mg} / \mathrm{str}$ \\
\hline
\end{tabular}
burned gas, as the Air-Fuel Ratio is regulated to its stoichiometric value in a SI engine.
}

$P_{i n t}$ and $T_{i n t}$ are measured by a sensor located in the intake manifold.

\section{A. Flow rate modeling}

To estimate the flow rate quantities given in (3), the model of aspirated air mass presented in [20] is used. In this model, $D_{a s p}$ is described as a function of the engine speed $N_{e}$, the manifold pressure $P_{i n t}$ and the intake and exhaust VVT actuators positions. Using the ideal gas law, this flow rate is dynamically related to the flow rate through the throttle as

$$
D_{t h r}=D_{a s p}\left(N_{e}, P_{i n t}, V V T\right)+\frac{V_{i n t}}{r T_{i n t}} \dot{P}_{i n t}
$$

where $r=r_{a i r}=r_{b g}$ is the common ideal gas constant.

\section{B. Transport delay characterization}

Equation (2) implicitly determines the transport delay according to the gas speed along the intake line, which is not measured in practice. Nevertheless, one can again exploit the ideal gas equation to relate this speed to current thermodynamical conditions and mass flow rates. In other words, this transport delay equation can be reformulated as

$$
V_{P}=\int_{t-\tau(t)}^{t} \frac{r T(s)}{P(s)}\left(D_{\text {air }}(s)+D_{\text {egr }}(s)\right) d s
$$

where $V_{P}$ is the total pipe volume and $T, P$ the current temperature and pressure values.

Observing the engine scheme depicted in Fig. 1, one can divide the intake line into three main sections with three respective transport delays :

- downstream the EGR valve to the compressor. In this portion, atmospheric conditions are used

$$
V_{3}=\int_{t-\tau_{3}(t)}^{t} \frac{r T_{a t m}}{P_{\text {atm }}}\left[D_{\text {air }}(s)+D_{\text {egr }}(s)\right] d s
$$

- downstream the compressor to the intercooler. The current pressure and temperature are measured

$$
V_{2}=\int_{t-\tau_{3}(t)-\tau_{2}(t)}^{t-\tau_{3}(t)} \frac{r T_{d c}}{P_{d c}}\left[D_{a i r}(s)+D_{e g r}(s)\right] d s
$$


- downstream the intercooler to the intake manifold. Classically, the temperature in this portion is assumed to be equal to the intake manifold temperature and the pressure to be the same than the one downstream the compressor. Namely,

$$
V_{1}=\int_{t-\tau_{3}(t)-\tau_{2}(t)-\tau_{1}(t)}^{t-\tau_{3}(t)-\tau_{2}(t)} \frac{r T_{\text {int }}}{P_{d c}}\left[D_{\text {air }}(s)+D_{\text {egr }}(s)\right] d s
$$

In practice, the intermediate volumes $V_{1}, V_{2}$ and $V_{3}$ are known. Then, one can calculate the delay in a very straightforward manner, inverting one after the other (5), (6) and finally (7), to obtain $\tau(t)=\tau_{1}(t)+\tau_{2}(t)+\tau_{3}(t)$, like it is done in [26] in a process context.

This delay calculation methodology has been validated on a reference high frequency simulator developed on a AMESim $^{T M}$ platform (commonly used for control engine purposes [12]) and presented in [19]. Fig 2 pictures a particular example of delay variation, corresponding to a tip-in occurring at constant engine speed $(1200 \mathrm{rpm})$. For a low torque request (before $2.25 \mathrm{~s}$ ), the air mass flow rate is also quite low, which results into a significant transport delay value (around $1.35 \mathrm{~s}$ ). During tip-in, the air mass flow substantially increases, which results into a much lower delay value (around $0.3 \mathrm{~s}$ ). Fig 2 shows the accuracy of the proposed delay modeling.

The control objective is to have system (3) to track a smooth trajectory $x^{r}(t)$. To do so, we aim at developing a prediction-based control law taking advantage of the knowledge of the implicit delay variation law (5)-(7). With this aim in view, we assume in the following that the intake burned gas rate is measured, which is not the case in a commercialline engine. This point is briefly discussed in Section IV. We now detail the design of a tailored prediction-based control law.

\section{CONTROL DESIGN FOR LINEAR TIME-VARYING INPUT-DELAY SYSTEMS}

In this section, we focus on the design of a predictionbased control law for linear time-varying input-delay systems. Therefore, we consider in this section a linear (potentially unstable) plant

$$
\dot{X}(t)=A X(t)+B U(t-\tau(t))
$$

where $X \in \mathbb{R}^{n}$ and $U$ is scalar. We assume that the pair $(A, B)$ is controllable and $\tau: \mathbb{R}_{+} \rightarrow[\underline{\tau}, \bar{\tau}]$ is a bounded and timedifferentiable function.

We also consider a time-varying reference $\left(X^{r}(t), U^{r}(t)\right)$ for the corresponding delay-free system, i.e. such that

$$
\dot{X}^{r}(t)=A X^{r}(t)+B U^{r}(t)
$$

and introduce the tracking error variables $\tilde{X}(t)=X(t)-X^{r}(t)$ and $\tilde{U}(t)=U(t)-U^{r}(t+\tau(t))$.
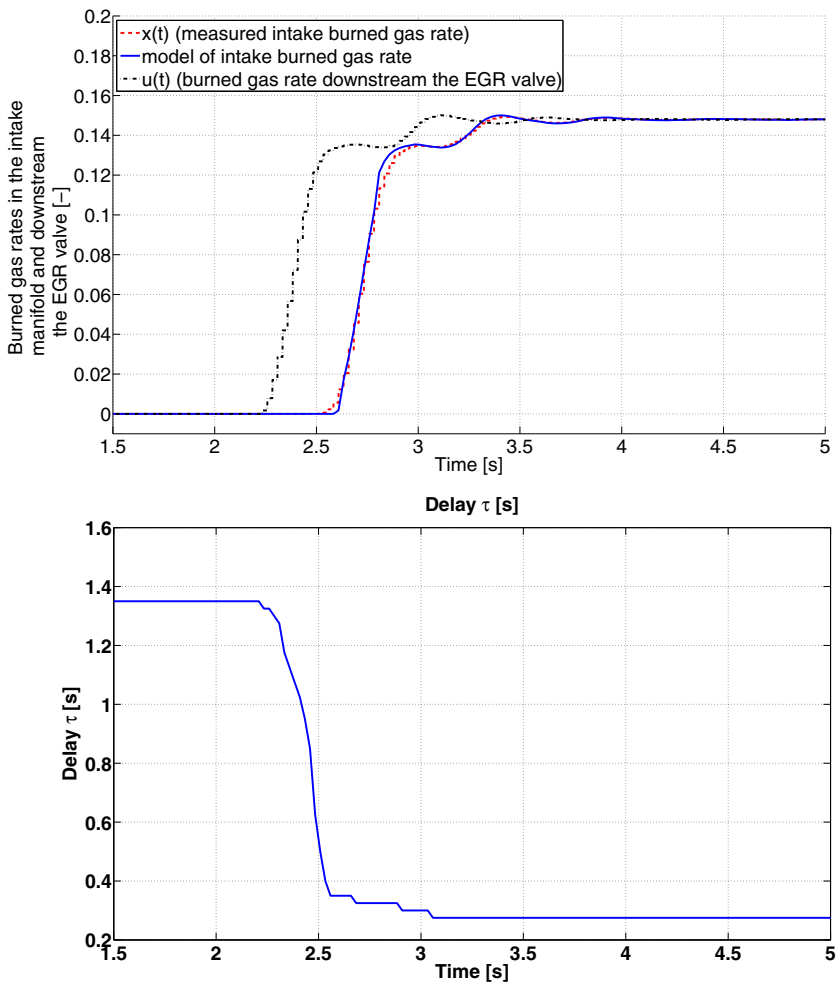

Fig. 2. Torque request transient (step from 20 to $200 \mathrm{Nm}$ ) for a constant engine speed of $1200 \mathrm{rpm}$. The above plot compares, for a given input $u(t)$, the intake burned gas rate $x$ (in red, dotted) with the model (1)-(3) (in blue). The model uses the delay value pictured in the above plot and obtained while inverting (5)-(7).

Theorem 1: Consider the closed-loop system consisting in the plant (8) and the control law

$$
\begin{aligned}
U(t)= & U^{r}(t+\tau(t))+K\left[e^{A \tau(t)} \tilde{X}(t)\right. \\
& \left.+\int_{t-\tau(t)}^{t} e^{A(t-s)} B\left[U(s)-U^{r}(s+\tau(t))\right] d s\right]
\end{aligned}
$$

where the vector $K$ is chosen such that $A+B K$ is Hurwitz. There exists $\left.\delta^{*} \in\right] 0,1[$ such that, provided

$$
\forall t \geq 0, \quad|\dot{\tau}(t)|<\delta^{*}
$$

the state tracking error $\tilde{X}(t)$ exponentially converges toward 0 when $t \rightarrow \infty$.

Control law (9) is directly inspired by the constant delay case, calculating the state prediction over a time window of varying length $D(t)$. Of course, exact compensation of the delay is not achieved with this controller. To do so, one would need to consider a time window of which length would match exactly the value of the future delay, as it is made in $[17]^{3}$. In other words, this requires to be able to predict the

\footnotetext{
${ }^{3}$ In details, defining the delay operator $\phi(t)=t-D(t)$ and assuming that its inverse exists and is available, exact delay-compensation is obtained with the feedback law $U(t)=K X\left(\phi^{-1}(t)\right)$ where the prediction can be written as $X\left(\phi^{-1}(t)\right)=\left[e^{A\left(\phi^{-1}(t)-t\right)}+\int_{t}^{\phi^{-1}(t)} e^{A\left(\phi^{-1}(t)-s\right)} B U(\phi(s)) d s\right]$
} 
future variation of the delay, which is not always practically achievable and then implementable.

In this context, (10) can be interpreted as a condition for robust compensation achievement. Namely, if the delay varies sufficiently slowly, its current value $D(t)$ used for prediction will be close enough to its future values, and the corresponding prediction will be accurate enough to ensure the stabilization of the plant.

Interestingly, the exact same condition is stated in [30], where the delay is also assumed to be time-differentiable. Yet, the approach designed in [30] differs from ours in the way that a constant average delay value is used for control ${ }^{4}$, which should naturally result into poorer performance than the proposed one.

Finally, an expression of the bound $\delta^{*}$ is provided in the following proof. Of course, as it results from a Lyapunov analysis, this value is extremely conservative, as the above simulations emphasize it. Nevertheless, it leads to the conclusion, at least according to the Lyapunov proof, that the faster the dynamics of the system is, the smaller this bound will be ${ }^{5}$. We now detail this proof.

Proof In the following, we use the Lyapunov tools introduced in [15] to analyze the stability of input time-delay systems and which are based on a backstepping transformation of a certain actuator state defined for constant delays ${ }^{6}$.

First, to extend them to the time-varying delay case, we introduce the distributed input $u(x, t)=U(t+D(t)(x-$ 1)), $x \in[0,1]$, which enables to rewrite plant (8) as

$$
\left\{\begin{aligned}
\dot{X}(t) & =A X(t)+B u(0, t) \\
D(t) u_{t}(x, t) & =u_{x}(x, t)+\dot{D}(t)(x-1) u_{x}(x, t) \\
u(1, t) & =U(t)
\end{aligned}\right.
$$

In details, the input delay is now represented as a coupling with a transport partial differential equation (PDE) driven by the input and where the convection speed varies both with space and time.

Further, we introduce an alternative control reference $V^{r}(t)=U^{r}(t+\tau(t))$ and define the corresponding distributed reference $v^{r}(x, t)=V^{r}(t+\tau(t)(x-1))$. Following the mentioned approach, we now consider the following backstepping transformation, based on the control tracking errors $e(x, t)=u(x, t)-v^{r}(x, t)$,

$$
w(x, t)=e(x, t)-\tau(t) K \int_{0}^{x} e^{A \tau(t) x} B e(y, t) d y-K e^{A \tau(t) x} \tilde{X}(t)
$$

This transformation enables to rewrite the error plant corre-

\footnotetext{
${ }^{4}$ More precisely, the control law is designed after a reduction model using this average value.

${ }^{5}$ In details, the constant $M_{1}, M_{2}$ and $M_{3}$ introduced below are then larger, which results into a smaller value of $\delta^{*}$.

${ }^{6}$ This transformation is made to convert the plant $\dot{X}(t)=A X(t)+B u(0, t)$, $\tau u_{t}(x, t)=u_{x}(x, t)$ with the boundary condition $u(1, t)=U(t)$ into the target system $\dot{X}(t)=(A+B K) X(t)+B w(0, t), \quad \tau w_{t}(x, t)=w_{x}(x, t)$ with the boundary condition $w(1, t)=0$.
}

sponding to (8)-(9) as

$$
\begin{aligned}
\dot{\tilde{X}}(t) & =(A+B K) \tilde{X}(t)+B w(0, t) \\
\tau(t) w_{t}(x, t) & =w_{x}(x, t)-\tau(t) \dot{\tau}(t) f(x, t) \\
w(1, t) & =0
\end{aligned}
$$

where the function $f$ can be expressed as

$$
\begin{aligned}
f(x, t)= & \frac{1-x}{\tau(t)} e_{x}(x, t)+K A x e^{A \tau(t) x} \tilde{X}(t) \\
& +K \int_{0}^{x} e^{A \tau(t)(x-y)} B e_{x}(y, t) d y \\
& +K \int_{0}^{x}(I+A \tau(t)(x-y)) e^{A \tau(t)(x-y)} B e(y, t) d y
\end{aligned}
$$

In the Lyapunov analysis, we also need the governing dynamical equation of the spatial derivative of the transformed distributed input

$$
\begin{aligned}
\tau(t) w_{x t}(x, t) & =w_{x x}(x, t)-\tau(t) \dot{\tau}(t) f_{x}(x, t) \\
w(1, t) & =\tau(t) \dot{\tau}(t) f(1, t)
\end{aligned}
$$

We now introduce the following Lyapunov-Krasovskii functional

$$
\begin{aligned}
V(t)= & \tilde{X}(t)^{T} P \tilde{X}(t)+b_{1} \tau(t) \int_{0}^{1}(1+x) w(x, t)^{2} d x \\
& +b_{2} \tau(t) \int_{0}^{1}(1+x) w_{x}(x, t)^{2} d x
\end{aligned}
$$

where the positive symmetric matrix $P$ satisfies the Lyapunov equation $P(A+B K)+(A+B K)^{T} P=-Q$, where $Q$ is a given definite positive symmetric matrix. Taking a time-derivative of the $V$, one can obtain with some integrations by parts

$$
\begin{aligned}
& \dot{V}(t)=-\tilde{X}(t)^{T} Q \tilde{X}(t)+2 \tilde{X}(t)^{T} P B w(0, t)-b_{1}\|w(t)\|^{2} \\
& -b_{1} w(0, t)^{2}-b_{2}\left\|w_{x}(t)\right\|^{2}+2 b_{2} w_{x}(1, t)^{2}-b_{2} w_{x}(0, t)^{2} \\
& -2 b_{1} \tau(t) \dot{\tau}(t) \int_{0}^{1}(1+x) w(x, t) f(x, t) d x \\
& -2 b_{2} \tau(t) \dot{\tau}(t) \int_{0}^{1}(1+x) w_{x}(x, t) f_{x}(x, t) d x \\
& +\dot{\tau}(t)\left[b_{1} \int_{0}^{1}(1+x) w(x, t)^{2} d x+b_{2} \int_{0}^{1}(1+x) w_{x}(x, t)^{2} d x\right] \\
& \leq-\frac{\lambda_{\min }(Q)}{2}|\tilde{X}(t)|^{2}-\left(b_{1}-\frac{2|P B|^{2}}{\lambda_{\min }(Q)}\right) w(0, t)^{2} \\
& -b_{1}\|w(t)\|^{2}-b_{2}\left\|w_{x}(t)\right\|^{2}-b_{2} w_{x}(0, t)^{2}+2 b_{2} w_{x}(1, t)^{2} \\
& +2 b_{1} \tau(t)|\dot{\tau}(t)|\left|\int_{0}^{1}(1+x) w(x, t) f(x, t) d x\right| \\
& +2 b_{2} \tau(t)|\dot{\tau}(t)|\left|\int_{0}^{1}(1+x) w_{x}(x, t) f_{x}(x, t) d x\right| \\
& +2|\dot{\tau}(t)|\left(b_{1}\|w(t)\|^{2}+b_{2}\left\|w_{x}(t)\right\|^{2}\right)
\end{aligned}
$$

Considering the inverse transformation of (11) which satisfies the following Volterra integral equation of the second kind

$$
\begin{aligned}
e(x, t)= & w(x, t)+K e^{(A+B K) \tau(t) x} \tilde{X}(t) \\
& +\tau(t) K \int_{0}^{x} e^{(A+B K) \tau(t)(x-y)} B w(y, t) d y
\end{aligned}
$$




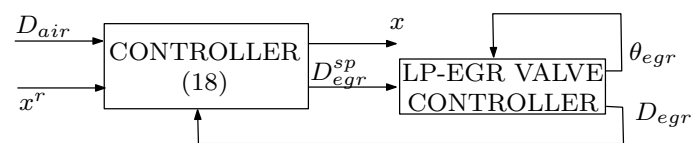

Fig. 3. Proposed controller architecture. The low-level EGR-valve controller exploits the mass-flow rate model given in Appendix.

one can obtain, using Cauchy-Schwartz and Young's inequality, the existence of positive constants $M_{1}, M_{2}$ and $M_{3}$ such that

$$
\begin{aligned}
& 2 w_{x}(1, t)^{2} \\
& \leq M_{1}|\dot{\tau}(t)|^{2}\left(|\tilde{X}(t)|^{2}+\|w(t)\|^{2}+\left\|w_{x}(t)\right\|^{2}\right) \\
& 2 \tau(t)\left|\int_{0}^{1}(1+x) w(x, t) f(x, t) d x\right| \\
& \leq M_{2}\left(|\tilde{X}(t)|^{2}+\|w(t)\|^{2}+\left\|w_{x}(t)\right\|^{2}\right) \\
& 2 \tau(t)\left|\int_{0}^{1}(1+x) w_{x}(x, t) f_{x}(x, t) d x\right| \\
& \leq M_{3}\left(|\tilde{X}(t)|^{2}+\|w(t)\|^{2}+\left\|w_{x}(t)\right\|^{2}+w_{x}(0, t)^{2}\right)
\end{aligned}
$$

Using these inequalities and defining $V_{0}(t)=|\tilde{X}(t)|^{2}+$ $\|w(t)\|^{2}+\left\|w_{x}(t)\right\|^{2}$, on can get

$$
\begin{gathered}
\dot{V}(t) \leq-\frac{\lambda_{\min }(Q)}{2}|\tilde{X}(t)|^{2}-\left(b_{1}-\frac{2|P B|^{2}}{\lambda_{\min }(Q)}\right) w(0, t)^{2} \\
-b_{1}\|w(t)\|^{2}-b_{2}\left\|w_{x}(t)\right\|^{2}-b_{2}\left(1-M_{3}|\dot{\tau}(t)|\right) w_{x}(0, t)^{2} \\
+|\dot{\tau}(t)|\left(b_{2} M_{1}|\dot{\tau}(t)|+b_{1} M_{2}+b_{2} M_{3}+2 b_{1}+2 b_{2}\right) V_{0}(t)
\end{gathered}
$$

Consequently, by choosing $b_{1}>2|P B|^{2} / \lambda_{\min }(Q)$ and defining

$$
\delta^{*}=\min \left\{\frac{\min \left\{\lambda_{\min }(Q) / 2, b_{1}, b_{2}\right\}}{b_{2} M_{1}+B_{1} M_{2}+b_{2} M_{3}+2 b_{1}+2 b_{2}}, 1, \frac{1}{M_{3}}\right\}
$$

we obtain the existence of a constant $\mu>0$ such that, for $|\dot{\tau}(t)|<\delta^{*}$

$$
\forall t \geq 0, \quad \dot{V}(t) \leq-\mu V_{0}(t)
$$

Finally, observing that both

$$
\begin{array}{r}
\min \left\{\lambda_{\min }(P), b_{1} \underline{\tau}, b_{2} \underline{\tau}\right\} V_{0}(t)=\eta_{1} V_{0}(t) \leq V(t) \\
V(t) \leq \max \left\{\lambda_{\max }(P), 2 b_{1} \bar{\tau}, 2 b_{2} \bar{\tau}\right\} V_{0}(t)=\eta_{2} V_{0}(t)
\end{array}
$$

one can deduce that

$$
\forall t \geq 0, \quad V_{0}(t) \leq \frac{\eta_{2}}{\eta_{1}} V_{0}(0) e^{-\frac{\mu}{\eta_{2}} t}
$$

This concludes the proof.

\section{Application to intake BURned gas RATE REGULATION AND SIMULATION RESULTS}

A direct application of Theorem 1 to the considered dynamics (3) yields the following choice for the controller

$$
\left\{\begin{array}{l}
D_{\text {egr }}(t)=D_{\text {air }}(t) \frac{u(t)}{1-u(t)} \\
u(t)=u^{r}(t+\tau(t))-k\left[e^{-\alpha D_{a s p} \tau(t)}\left[x(t)-x^{r}(t)\right]\right. \\
+\alpha D_{t h r} \int_{t-\tau(t)}^{t} e^{\left.-(t-s) \alpha D_{a s p}\left[u(t)-u^{r}(s+\tau(t))\right] d s\right]}
\end{array}\right.
$$

In practice, this control law is saturated as it is realized with the EGR-valve, which is a limited actuator ${ }^{7}$. The controller architecture is summarized in Fig. 3.

Fig. 4 presents simulation results corresponding to a given operating point (constant engine speed $N_{e}=1200 \mathrm{rpm}$ and torque request $T_{q}=120 \mathrm{Nm}$ ), for the considered reference intake burned gas rate depicted in the left-hand plot. The feedback gain has been chosen as $k=0.1$.

One can observe that, starting without external gas recirculation, i.e. with $x(0)=0$, the convergence is predictably achieved. The effect of the feedback term into (18) can be noticed at the beginning of the simulation, as the EGR mass flow rate overshoots aiming at decreasing the time response of the system. After that, the regulation is mainly achieved with the feedforward term.

Further, a few comments can be made about the achievement of the condition (10). A direct computation of the bound $\delta^{*}$ for the considered case leads to a scale of $10^{-2}$, which is extremely conservative and cannot be used in practice. Nevertheless, in Fig. 4, one can observe that the delay varies quite slowly, which is compliant with the spirit of this condition. More in details, taking a time-derivative of the implicit equation (2), the following expression of the delay derivative is obtained

$$
\dot{\tau}(t)=1-\frac{D_{\text {air }}(t)+D_{\text {egr }}(t)}{D_{\text {air }}(t-\tau)+D_{\text {egr }}(t-\tau)}
$$

Then, considering the mass flow rates pictured in Fig. 3, a first-order approximation leads to $\dot{\tau}(t) \approx 1-\frac{D_{\text {air }}(t)}{D_{\text {air }}(t-\tau)} \approx 0$ for the considered case. For a given operating point, expression (19) also indicates that the achievable EGR trajectories should not include at time $t$ frequencies above $1 / \tau(t)$.

These considerations yield to the conclusion that, for sufficiently slow variations of the air flow (which correspond to slow variations of the driver torque request), the proposed technique can be directly applied. However, the EGR trajectory generation seems arduous for substantial transient behavior, such as tip-ins. This is a direction of future work.

Finally, as the intake burned gas rate is usually unmeasured in commercial-line engines, upcoming works will also focus

\footnotetext{
${ }^{7}$ In details, this control law is transcribed into a EGR-valve position using the model given in Appendix A. This model exploits the measurement of the differential of pressure $\Delta P$ given by a dedicated sensor, represented in Fig. 1 . A further study of this model also reveals that the range of achievable EGR flow rate is mainly limited by this differential of pressure and, consequently, depends on the considered operating point.
} 

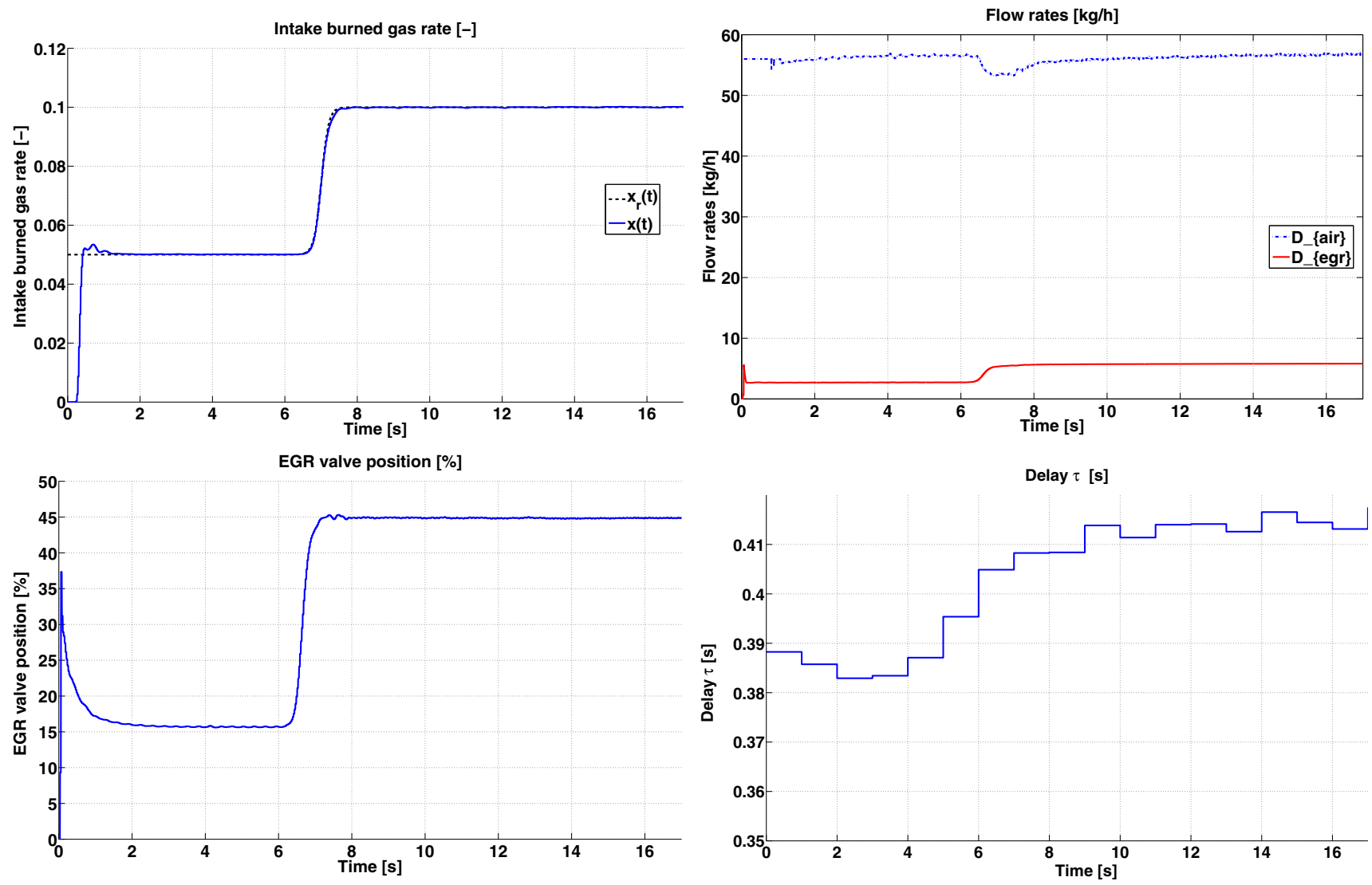

Fig. 4. Simulation results for constant engine speed $\left(N_{e}=1200 \mathrm{rpm}\right)$ and torque request $\left(T_{q}=120 \mathrm{Nm}\right)$. The reference trajectory of intake burned gas rate is pictured in the left-hand figure (dotted).

on the design of an estimate to feed the control law ${ }^{8}$.

\section{CONCLUSION AND PERSPECTIVES}

In this paper, a novel prediction-based control law for linear systems with time-varying input delay has been designed and applied to the trajectory tracking of the intake burned gas rate for SI engines. Simulation validation of the proposed model has been carried out on the AMESim ${ }^{T M}$ platform and control results highlight the merits of the proposed approach.

The next step is to validate both the proposed model and controller on experimental test-benches. Based on the simulation results, one can reasonably expect combustion performance improvements.

\footnotetext{
${ }^{8}$ One promising way could be to exploit the exhaust Air-Fuel Ratio measurement given by the lambda sensor (see Fig. 1) jointly with the history of the injected mass of fuel and an estimation of the exhaust transport delay, due to the location of the lambda sensor.
}

\section{APPENDIX}

\section{A. Mass flow rate through the EGR Valve}

The EGR mass flow rate can be assumed as sub-critical and modeled (see [11]) as

$$
\begin{aligned}
D_{\text {egr }} & =S_{\text {valve }} \psi\left(P_{u v}\right) \\
\psi\left(P_{u v}\right) & =\frac{P_{u v}}{\sqrt{R T_{e g r}}}\left(\frac{P_{a t m}}{P_{u v}}\right)^{1 / \gamma} \sqrt{\frac{2 \gamma}{\gamma-1}\left(1-\left(\frac{P_{a t m}}{P_{u v}}\right)^{\frac{\gamma-1}{\gamma}}\right)}
\end{aligned}
$$

where $S_{t h r}$ is the effective opening area of the throttle, $P_{u v}$ is the upstream valve pressure, measured obtained from the atmospheric pressure and the $\Delta P$-sensor measurements and $\gamma$ is the ratio of specific heat. The effective area is itself statically related to the angular position of the actuator.

In practice, an alternative linearized model may be needed to account for the potential low values of the differential of pressure $\Delta P$, which result into a pressure ratio $P_{a t m} / P_{u v}$ close to the unity.

\section{REFERENCES}

[1] M. Ammann, N. P. Fekete, L. Guzzella, and A.H. Glattfelder. Modelbased control of the VGT and EGR in a turbocharged commonrail Diesel engine: theory and passenger car implementation. $S A E$ transactions, 112(3):527-538, 2003. 
[2] Z. Artstein. Linear systems with delayed controls: a reduction. IEEE Transactions on Automatic Control, 27(4):869-879, 1982.

[3] D. Bresch-Pietri, J. Chauvin, and N. Petit. Adaptive backstepping controller for uncertain systems with unknown input time-delay. application to SI engines. In Proc. of the Conference on Decision and Control, 2010.

[4] D. Bresch-Pietri, J. Chauvin, and N. Petit. Output feedback control of time delay systems with adaptation of delay estimate. In Proc. of the IFAC World Congress, 2011.

[5] D. Bresch-Pietri and M. Krstic. Adaptive trajectory tracking despite unknown input delay and plant parameters. Automatica, 45(9):20742081, 2009.

[6] A. Cairns and H. Blaxill. The effects of combined internal and external exhaust gas recirculation on gasoline controlled auto-ignition. SAE Technical Paper, -:01-0133, 2005.

[7] L. Eriksson. Spark Advance Modeling and Control. PhD thesis, Linkoping University, May 1999.

[8] K. Gu and S. I. Niculescu. Survey on recent results in the stability and control of time-delay systems. Journal of Dynamic Systems, Measurement, and Control, 125:158, 2003.

[9] Q. L. Han and K. Gu. Stability of linear systems with time-varying delay: a generalized discretized lyapunov functional approach. Asian Journal of Control, 3(3):170-180, 2001.

[10] Y. He, Q. G. Wang, C. Lin, and M. Wu. Delay-range-dependent stability for systems with time-varying delay. Automatica, 43(2):371376, 2007.

[11] J. B. Heywood. Internal combustion engine fundamentals. McGrawHill New York, 1988.

[12] LMS IMAGINE. http://www.lmsintl.com.

[13] M. Jankovic. Recursive predictor design for linear systems with time delay. In American Control Conference, 2008, pages 4904-4909. IEEE, 2008.

[14] X. Jiang and Q. L. Han. New stability criteria for linear systems with interval time-varying delay. Automatica, 44(10):2680-2685, 2008.

[15] M. Krstic. Boundary control of PDEs: A course on backstepping designs. Society for Industrial and Applied Mathematics Philadelphia, PA, USA, 2008.

[16] M. Krstic. Lyapunov tools for predictor feedbacks for delay systems: Inverse optimality and robustness to delay mismatch. Automatica, 44(11):2930-2935, 2008.

[17] M. Krstic. Delay compensation for nonlinear, adaptive, and PDE systems. Birkhauser, 2009.

[18] M. Krstic and D. Bresch-Pietri. Delay-adaptive full-state predictor feedback for systems with unknown long actuator delay. In Proceedings of the 2009 conference on American Control Conference, pages 4500-4505, 2009.

[19] F. Le Berr, M. Miche, G. Le Solliec, F. A. Lafossas, and G. Colin. Modelling of a turbocharged SI engine with variable camshaft timing for engine control purposes. In SAE World Congress, volume number 2006-01-3264, 2006.

[20] T. Leroy, J. Chauvin, F. Le Berr, A. Duparchy, and G. Alix. Modeling fresh air charge and residual gas fraction on a dual independant variable valve timing SI engine. SAE International Journal of Engines, 1(1):627-635, 2009.

[21] T. Leroy, J. Chauvin, and N. Petit. Motion planning for experimental air path control of a variable-valve-timing spark ignition engine. Control Engineering Practice, 17(12):1432-1439, 2009.

[22] Y. Liu and L.-S. Hu. New stabilization method for linear systems with time-varying input delay. In American Control Conference, 2011.

[23] A. Manitius and A. Olbrot. Finite spectrum assignment problem for systems with delays. Automatic Control, IEEE Transactions on, 24(4):541-552, 2002.

[24] S. Mondié and W. Michiels. A safe implementation for finite spectrum assignment: robustness analysis. In Proc. of the 42nd IEEE Conference on Decision and Control (CDC2003), Hawaii, USA, 2003.

[25] Y. S. Moon, P. G. Park, and W. H. Kwon. Robust stabilization of uncertain input-delayed systems using reduction method. Automatica, 37(2):307-312, 2001.

[26] N. Petit, Y. Creff, and P. Rouchon. Motion planning for two classes of nonlinear systems with delays depending on the control. In Proceedings of the 37th IEEE Conference on Decision and Control, volume 1, pages 1007-1011. IEEE, 1998.

[27] J.-P. Richard. Time-delay systems: an overview of some recent advances and open problems. Automatica, 39(10):1667-1694, 2003.
[28] O. J. M. Smith. A controller to overcome dead time. ISA Journal, 6(2):28-33, 1959.

[29] M. J. Van Nieuwstadt, I. V. Kolmanovsky, P. E. Moraal, A. Stefanopoulou, and M. Jankovic. Egr-vgt control schemes: experimental comparison for a high-speed diesel engine. Control Systems Magazine, IEEE, 20(3):63-79, 2000.

[30] D. Yue and Q. L. Han. Delayed feedback control of uncertain systems with time-varying input delay. Automatica, 41(2):233-240, 2005.

[31] M. Zheng, G. T. Reader, and J. G. Hawley. Diesel engine exhaust gas recirculation-a review on advanced and novel concepts. Energy Conversion and Management, 45(6):883-900, 2004. 